\title{
Comparison of vascular endothelial growth factor inhibitors on macular oedema secondary central retinal vein occlusion
}

\author{
Kelvin Yi Chong Teo ${ }^{1,2} \cdot$ Aditi Mohla ${ }^{1,3} \cdot$ Chui Ming Gemmy Cheung $\mathbb{D}^{1,2,4}$
}

Received: 29 September 2019 / Accepted: 22 October 2019 / Published online: 26 November 2019

(c) The Royal College of Ophthalmologists 2019

The three vascular endothelial growth factor (VEGF) inhibitors in use: aflibercept, bevacizumab, and ranibizumab have been shown to be effective in treating retinal vein occlusion (RVO) related central macular oedema (CMO) [1-4] and other retina angiogenic disease like age-related macular degeneration and diabetic macular oedema. Unlike the DRCR.net protocol $\mathrm{T}$ which demonstrated better visual outcomes with aflibercept compared with the other agents for DMO [5], the Study of Comparative Treatments for Retinal Vein Occlusion (SCORE2) reported no difference between aflibercept and bevacizumab at 6 months for the treatment of CRVO-related CMO [6]. It is still unclear however, which of the three VEGF inhibitors provides best outcomes.

The Lucentis, Eylea, Avastin in Vein Occlusion (LEAVO) study is the first trial to compare the outcomes of treatment of CRVO-related CMO with the three available VEGF inhibitors. This was a non-inferiority study that randomly assigned 463 patients into treatment with aflibercept, ranibizumab or bevacizumab [7]. The primary outcome showed that bevacizumab was not non-inferior compared with ranibizumab while aflibercept was non-inferior but not superior to ranibizumab. This result supports current guidelines by the National Institute for Health and Care Excellence and the Royal College of Ophthalmologists which recommends the use of either ranibizumab or aflibercept for CRVO-related CMO.

Chui Ming Gemmy Cheung

gemmy.cheung.c.m@snec.com.sg

1 Singapore Eye Research Institute, Singapore National Eye Centre, Singapore, Singapore

2 Ophthalmology \& Visual Sciences Academic Clinical Program (Eye ACP), Duke-NUS Medical School, Singapore, Singapore

3 Department of Ophthalmology, Leeds Teaching Hospitals NHS Trust, Leeds, UK

4 Department of Ophthalmology, National University of Singapore, Singapore, Singapore
When selecting between ranibizumab and aflibercept managed with a pro-re-nata retreatment strategy, aflibercept was shown to save a mean of two injections over 100 weeks compared with ranibizumab, and more eyes achieved a central subfield thickness of $<320 \mu \mathrm{m}$ at both weeks 52 and 100 . These findings suggest that these two drugs have similar efficacy, with aflibercept having a slight advantage in terms of retreatment need. It would be of interest to assess whether these differences translate into significant long term impact on functional and quality of life outcomes.

Although bevacizumab was found to be not noninferior to ranibizumab, patients in this arm also experienced a significant visual acuity gain ( 9.8 letters). However, the lower limit of $95 \%$ confidence interval suggests the difference between the bevacizumab and ranibizumab arms may be as much as six letters, in favour of ranibizumab.

Thus, overall the LEAVO study findings suggest that ranibizumab or aflibercept should be used as first line treatment for CRVO-related CMO for best outcomes. However, in non-reimbursed settings outside of the National Health Service, the large price differential between agents make it difficult to justify advocating the use of these agents over bevacizumab. The preference and trends survey conducted by the American Society of Retina Specialist revealed that a majority of physicians in Asia and the United States use bevacizumab as first line treatment for CRVO-related CMO [8]. Ultimately, it is reassuring that treatment with any of the three VEGF inhibitor showed a significant improvement in mean vision at 100 days (12.5 for ranibizumab; 15.1 for aflibercept, and 9.8 for bevacizumab). The off-label use of bevacizumab for CRVO-related CMO is likely to continue in self-paid settings. Nonetheless, the LEAVO study has provided important information for counselling patients when bevacizumab is considered for cost-effectiveness.

\section{Compliance with ethical standards}

Conflict of interest The authors declare that they have no conflict of interest. 
Publisher's note Springer Nature remains neutral with regard to jurisdictional claims in published maps and institutional affiliations.

\section{References}

1. Brown DM, Campochiaro PA, Singh RP, Li Z, Gray S, Saroj N, et al. Ranibizumab for macular edema following central retinal vein occlusion: six-month primary end point results of a phase III study. Ophthalmology. 2010;117:1124-33.e1121.

2. Epstein DL, Algvere PV, von Wendt G, Seregard S, Kvanta A. Bevacizumab for macular edema in central retinal vein occlusion: a prospective, randomized, double-masked clinical study. Ophthalmology. 2012;119:1184-9.

3. Brown DM, Heier JS, Clark WL, Boyer DS, Vitti R, Berliner AJ, et al. Intravitreal aflibercept injection for macular edema secondary to central retinal vein occlusion: 1-year results from the phase 3 COPERNICUS study. Am J Ophthalmol. 2013;155:429-37.e427.

4. Korobelnik JF, Holz FG, Roider J, Ogura Y, Simader C, SchmidtErfurth U, et al. Intravitreal aflibercept injection for macular edema resulting from central retinal vein occlusion: one-year results of the phase 3 GALILEO Study. Ophthalmology. 2014;121:202-8.

5. Wells JA, Glassman AR, Ayala AR, Jampol LM, Bressler NM, Bressler SB, et al. Aflibercept, bevacizumab, or ranibizumab for diabetic macular edema: two-year results from a comparative effectiveness randomized clinical trial. Ophthalmology. 2016;123: 1351-9.

6. Scott IU, VanVeldhuisen PC, Ip MS, Blodi BA, Oden NL, Awh $\mathrm{CC}$, et al. Effect of bevacizumab vs aflibercept on visual acuity among patients with macular edema due to central retinal vein occlusion: the SCORE2 randomized clinical trial. JAMA. 2017; 317:2072-87.

7. Hykin P, Prevost AT, Vasconcelos JC, Murphy C, Kelly J, Ramu J, et al. Clinical effectiveness of intravitreal therapy with ranibizumab vs aflibercept vs bevacizumab for macular edema secondary to central retinal vein occlusion: a randomized clinical trial. JAMA Ophthalmol. 2019. https://doi.org/10.1001/jama ophthalmol.2019.3305. [Epub ahead of print].

8. Stone TW, editor. ASRS 2017 preferences and trends membership survey. Chicago, IL: American Society of Retina Specialists; 2018. 